\title{
Tapered Flow Diverters in the Treatment of Intracranial Aneurysms
}

\author{
Ahmet PEKER ${ }^{1}$, Erol AKGUL ${ }^{2}$, Ergun DAGLIOGLU ${ }^{3}$, Ilkay AKMANGIT ${ }^{4}$, Tunca KAYA ${ }^{4}$, Deniz BELEN ${ }^{3}$, Anil ARAT \\ ${ }^{1}$ Hacettepe University, School of Medicine, Department of Radiology, Ankara, Turkey \\ ${ }^{2}$ Cukurova University, School of Medicine, Department of Radiology, Adana, Turkey \\ ${ }^{3}$ Numune Education and Research Hospital, Neurosurgery Clinic, Ankara, Turkey \\ ${ }^{4}$ Numune Education and Research Hospital, Radiology Clinic, Ankara, Turkey
}

\section{ABSTRACT}

AIM: To report the initial experience of cerebral aneurysm treatment with Tapered flow diverters (TFDs).

MATERIAL and METHODS: Thirty patients with 34 aneurysms underwent cerebral aneurysm treatment with TFD (Silk, Balt, Montmorency, France) between March 2011 and March 2016. Procedural findings, complications, clinical and imaging follow-up were assessed retrospectively.

RESULTS: The patients' mean age was $48 \pm 14.5$ years (range, 16-74; 25 females). Aneurysms size ranged from 3 to $35 \mathrm{~mm}$ with an average diameter of $13.9 \pm 8.8 \mathrm{~mm}$ and a median diameter of $10.5 \mathrm{~mm}$. Technical success rate was $96.6 \%$. Technically, deployment of the device was similar to the non-tapered version and subjectively, it appeared to be easier in the paraophthalmic segment. Permanent morbidity and mortality rates secondary to the procedure were $0 \%$. On clinical follow-up ( 29 patients, mean $9.3 \pm 9.1$ months) there were no clinical untoward events. Imaging follow-up was at or after 6 months (20 patients, mean $12.3 \pm 10$ months). Angiographic occlusion rate was $80 \%$.

CONCLUSION: TFD is safe to use and effective for the treatment of intracranial aneurysms in this series. The occlusion rate is higher with respect to the previous reports and experience using the non-tapered version. Maintenance of porosity at the transition zone may be the factor underlying the higher occlusion rate. TFD may be preferred especially for arterial segments aneurysms where there is considerable discrepancy in size between the distal and proximal parent artery.

KEYWORDS: Flow diverter, Endovascular treatment, Intracranial aneurysms, Tapered devices

\section{INTRODUCTION}

$\Gamma$

Tapered flow diverters (TFD, Silk, Balt, Montmorency, France) are manufactured for the treatment of intracranial aneurysms in which there is a discrepancy between the diameters of the distal and proximal landing zones of the device on the parent artery.

We present the initial findings of cerebral aneurysm treatment with TFDs.

\section{MATERIAL and METHODS}

All patients treated by TFD between March 2011 and March 2016 by 2 endovascular teams were analyzed retrospectively. The treatments were performed in accordance with the previously published endovascular techniques utilizing the Silk device (Balt, Montmorency, France) under general anesthesia and systemic heparinization (10). At least five days before the procedure, patients started to use $75 \mathrm{mg}$ clopidogrel and 300 $\mathrm{mg}$ acetyl salysilic acid (ASA). The patients that underwent urgent surgery due to subarachnoid hemorrhage $(\mathrm{SAH})$ were 
given a loading dose of $600 \mathrm{mg}$ clopidogrel shortly before the endovascular procedure. Post-operatively all patients continued with $300 \mathrm{mg}$ ASA and $75 \mathrm{mg}$ clopidogrel once daily for 6 months for the maintenance treatment. In general terms after placement of guiding sheath via transfemoral route, distal access catheter was advanced to a secure point in the parent artery. For delivery of TFD, either the Vasco+21 or Copernic 2L balloon catheter (Balt Extrusion, Montmorency, France) were used. When we used a Copernic $2 \mathrm{~L}$ balloon catheter, wall apposition was provided with angioplasty, without an exchange manoeuvre. Patients were scheduled for angiography control (computed tomographic angiography or magnetic resonance angiography or digital subtraction angiography) at the post-operative sixth month. After this angiography control, treatment was continued with $300 \mathrm{mg}$ ASA alone. If the patient had more than one aneurysm on the same segment treated by a single TFD, only the largest aneurysm of that segment was included in the final clinical and radiologic evaluation. For the determination of safety of the device, all patients in whom placement of a tapered device was attempted were included in the final analysis. In accordance with the current literature, for the evaluation of efficacy, only patients with an angiographic follow-up (at least 6 months) were included since it is known that the occlusion of aneurysms with flow diversion is not an immediate process. Procedural findings, complications, clinical and imaging follow-up were assessed retrospectively.

Categorical variables were assessed by the Chi-square test or Fisher's Exact test, where applicable. The Shapiro-Wilk test was used to test normality assumptions of the continuous variables. Continuous variables were evaluated by Student's $t$ test where appropriate. $P$ value less than 0.05 was considered as significant.

\section{口 RESULTS}

Thirty patients with 34 aneurysms underwent cerebral aneurysm treatment with TFD between March 2011 and February 2016 by 2 endovascular teams. Device deployment was successful in all patients but one, yielding a technical success rate of $96.6 \%$. Deployment technique was similar to the non-tapered version and subjectively, it appeared to be easier in the paraophthalmic segment. The patients' mean age was $48 \pm 14.5$ years (range, 16-74; 25 females). Aneurysm location was as follows: 17 paraophthalmic artery origin (transitional segment/superior hypophysial artery/ophthalmic artery/carotid cave aneurysms), 10 supraclinoid internal carotid artery (posterior communicating artery/choroidal segments, an example is provided in Figure 1A-D), 2 cavernous carotid artery, 2 middle cerebral artery, 1 intracranial vertebral artery, 1 posterior inferior cerebellar artery and 1 basilar artery. Aneurysm size ranged from 3 to $35 \mathrm{~mm}$ with an average diameter of $13.9 \pm 8.8 \mathrm{~mm}$ and a median diameter of 10.5 $\mathrm{mm}$. Twenty-eight aneurysms were saccular, 6 were fusiform. The 3 posterior circulation aneurysms were of fusiform type. One patient presented during the acute phase of SAH and 4 patients presented with residual/recurrent aneurysms. Previous treatments in these patients included stent-assisted coiling (2 patients), flow diverter placement (2 patients), coiling
(2 patients) and clipping (1 patient). Additional devices were used during treatment in 5 patients. Additional Silk devices were used in 1 patient to increase neck coverage in an acutely ruptured blister-like aneurysm. In 1 patient presenting with a residual giant cavernous aneurysm previously treated by flow diversion, the tapered device was not long enough to cover the $30 \mathrm{~mm}$ aneurysm neck and a second flow diverter was placed inferior to the tapered device to be able to cover the whole neck from the healthy arterial segment distally to the healthy artery proximally. Similarly, in 3 patients the aneurysm was too large to be covered by a single flow diverter, and consequently a $35 \mathrm{~mm}$ or $50 \mathrm{~mm}$ long scaffolding stent (Leo stent, Balt, Montmorency, France) was used to be able to place the TFD accurately without migration into the aneurysm.

In terms of age, sex, aneurysm size and aneurysm location (anterior versus posterior circulation) and rate of use of adjunctive stent devices there was no statistically significant difference as compared to the previously reported series (11) the 2 patients included in both series were excluded from the first series and included in the current one for the statistical analysis. In one patient there was transient worsening of optic nerve compression that was present before the procedure and in a second patient a groin hematoma occurred after the procedure and was managed without surgery. Permanent morbidity and mortality rates secondary to the procedure were $0 \%$. One patient presented with worsening of previously existing hydrocephalus 2 months after the procedure. The attending surgical team refrained from a shunting procedure as the patient was on dual anti-platelet therapy. The patient's clinical status gradually worsened and finally the patient died secondary to consequences of increased intracranial pressure. On clinical follow-up (29 patients in whom TFD placement was successful, mean $9.3 \pm 9.1$ months), there were no other clinical events. Imaging follow-up at or after 6 months (20 patients, mean $12.3 \pm 10$ months) revealed an angiographic occlusion rate of $80 \%$. Asymptomatic stent occlusion was noted in one patient and asymptomatic parent artery stenosis was present in 3 cases.

\section{- DISCUSSION}

Flow diversion has revolutionized the treatment of intracranial aneurysms but problems related to residual or recurrent aneurysms still remain. An approximately 9.63 times risk for aneurysmal remnants was found for aneurysms greater than $13 \mathrm{~mm}$ in largest size as compared to smaller aneurysms (11), and a higher rate for residual aneurysms was found on followup for aneurysms from which large side-branches originated (17).

In wide-neck or fusiform aneurysms, there is frequently a discrepancy between the proximal and distal landing zones of the stent and in dissecting aneurysms this discrepancy may be more pronounced at the affected segment (in form of a stenosis). The discrepancy between the size of the landing zones will result in undersizing or oversizing of the device at either the proximal landing zone or the distal one based on what the operator chooses as his/her reference for the sizing of the flow diverter (16). Inevitably, there will be a conical transitional zone (TZ) between the fully expanded or even over expanded 


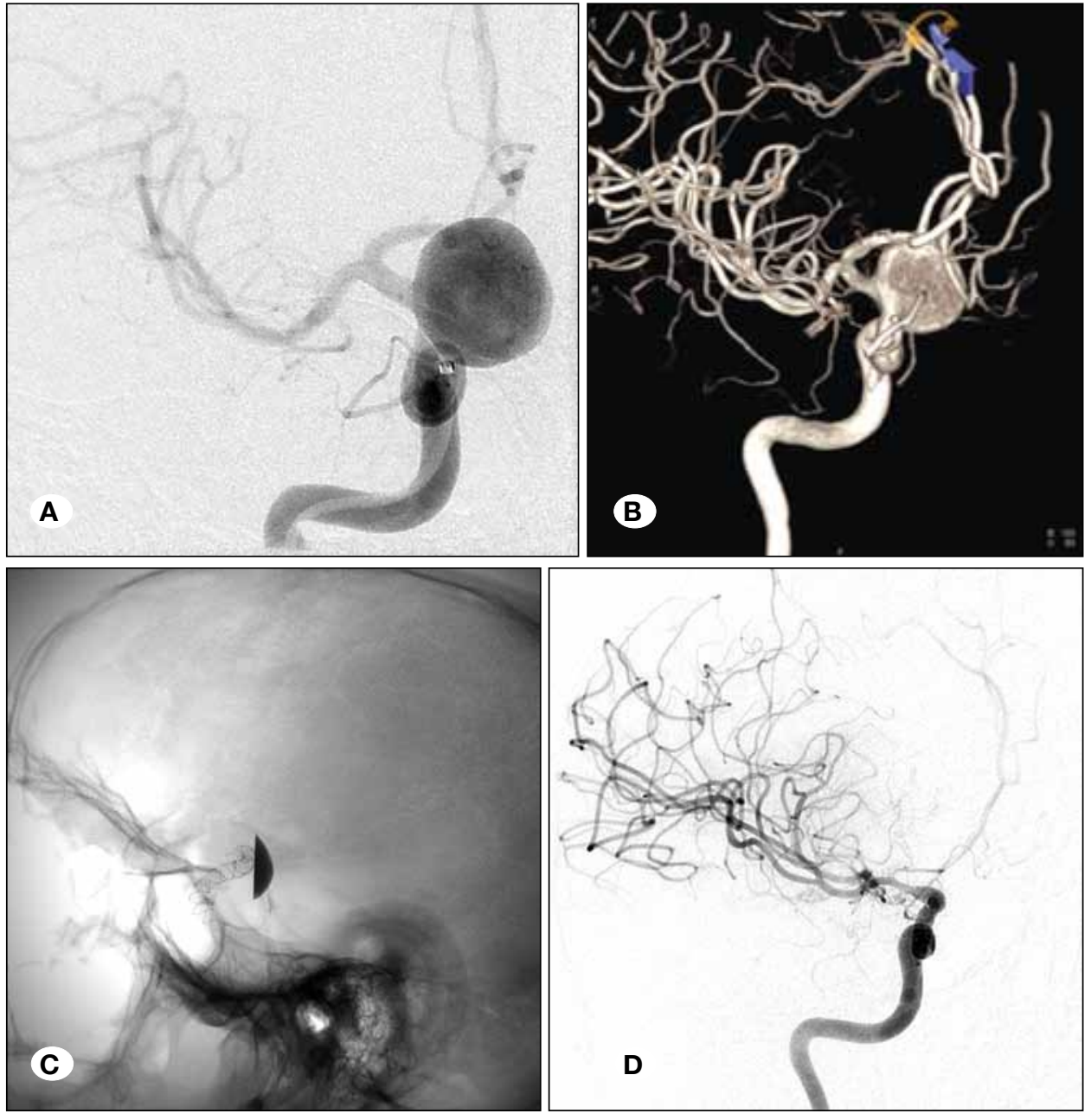

Figure 1: Pre-procedure digital subtraction angiogram in AP view (A), and 3-dimensional reconstruction of the same radiographic view $\mathbf{B}$ ) demonstrates $20 \mathrm{~mm}$ supraclinoid aneurysm. (C) Native view in lateral projection obtained after placement of a single Tapered flow diverter (TFD) shows stagnation of contrast material in the aneurysm at the end of the procedure. (D) Follow-up angiogram obtained at 12 months reveals no evidence of a residual aneurysm, moderate amount of neointimal hyperplasia at the distal end of the TFD, and interval diminution of the A1 segment of the ipsilateral anterior cerebral artery which was filling predominantly from the left side through the anterior communication artery. segment crossing the aneurysm and the relatively constrained distal or proximal landing zones (9). In this transitional zone, metal coverage is lower, porosity is higher and on follow-up neointima formation is poor $(14,16)$. In other words, as there is a direct relation between lower porosity of the 'free segment of the stent' (FSS) facing the aneurysm cavity and success of aneurysm occlusion $(6,7)$, the increased porosity at TZ will result in sustained leak into the aneurysm and increase the risk of residual aneurysms after flow diversion $(2,9,13,15$, 16). Focal zones of low porosity and residual flow specifically occur in curved segments or wide-neck aneurysms due to the deformation of the FD $(8,13)$. This deformation at the TZ is a potential cause of failure of flow diversion in wide-neck curved and/or endwall aneurysms $(2,6-8,13)$.

Although compression and "inflation" have been used to segmentally increase the mesh density of braided endovascular prostheses $(4,10)$ most often these techniques are insufficient to overcome the low porosity in the transition zone $(2,13,15)$. Likewise, using FDs with higher pore density or telescoping the FD devices was not sufficient to reliably occlude giant side wall or bifurcation aneurysms (5). In this respect TZ appears to be an unavoidable cause of treatment failure.
Some authors suggest that conical placement of FDs at the proximal and distal landing zones (e.g. placement of FDs of subsequently increasing diameters telescopically, in an overlapping fashion) will increase the metal coverage and counteract the untoward effects of the high porosity at the TZ $(15,16)$. However multi-device FD constructs may result in perforator artery injuries or increase the cost or increase the complexity of the procedure (10) in comparison to the TFD.

TFDs have been manufactured in a conical shape specifically for arterial segments with discrepant landing zones, such as the carotid siphon (1), and also for irregular arterial segments. Although these devices have been available clinically since 2010 , there is currently no report related to these devices in the literature. With these devices, in a manner similar to the conical placement of FDs, the porosity change at TZ is expected to be lower than the conventional FDs (personal communication, Nicolas Plowiecki, inventor of TFD, WINABC Meeting, Val d'Isere, Cedex, France). In our series we were able to achieve an approximately 80 percent occlusion rate. This result compares favorably to results of the previous reports utilizing the non-tapered version of these stents $(3,12)$. Considering the larger size of the aneurysms (mean size larger than $13 \mathrm{~mm}$ ) in our series, as a matter of fact, our occlusion 
rate corresponds to a higher occlusion rate and efficacy of the TFD device.

Optimal sizing of FDs is not only important for occlusion rates on follow-up but it is also critical during deployment of the device. More specifically, when the device is chosen according to the arterial segment with lower diameter, overexpansion occurs in the larger arterial segment and this is associated with severe shortening as well as suboptimal apposition and even migration if re-crossing of the device with the deployment catheter is necessary. When the choice is made according to the larger diameter of the parent artery on the other hand, difficulties in stent deployment and hourglass formation at bends are encountered (10). Based on our limited experience, technically, TFDs are more easily deployed in arterial segments with size discrepancy.

TFDs were not associated with an increased rate of adverse events in this study. As a matter of fact, device-related mortality/ morbidity and procedure related mortality/permanent morbidity was $0 \%$, there were high rate technical success $96.6 \%$. The fatal case was secondary to reluctance of ventriculoperitoneal shunt placement under anti-platelet therapy so it is not related to the device used, nor is the procedure directly associated with this outcome. Angiographically the aneurysm of this patient was not filling. There was no suggestion of an increased thromboembolic risk associated with the conical design. In addition, subjective ease of deployment and small delivery system (Vasco 21 microcatheter, OD: $2.7 \mathrm{~F}$ and ID: 0024 inches) (Balt, Montmorency, France) may potentially decrease hemorrhagic events (such as perforations or dissections associated to the distal wire) secondary to easier access and decreased manipulation of the device.

\section{- CONCLUSION}

TFD is safe to use and effective for the treatment of intracranial aneurysms in this series. The occlusion rate is higher with respect to the previous reports using the non-tapered version. TFD may be preferred especially for aneurysms in the paraophthalmic region where there is considerable difference in size between the distal and proximal parent artery.

\section{REFERENCES}

1. Arat $\mathrm{YO}$, Arat A, Aydin K: Angiographic morphometry of internal carotid artery circulation in Turkish children. Turk Neurosurg 25: 608-616, 2015

2. Bing F, Darsaut TE, Salazkin I, Makoyeva A, Gevry G, Raymond $\mathrm{J}$ : Stents and flow diverters in the treatment of aneurysms: Device deformation in vivo may alter porosity and impact efficacy. Neuroradiology 55: 85-92, 2013

3. Buyukkaya R, Kocaeli H, Yildirim N, Cebeci H, Erdogan C, Hakyemez B: Treatment of complex intracranial aneurysms using flow-diverting silk stents. An analysis of 32 consecutive patients. Interv Neuroradiol 20:729-735, 2014

4. Daglioglu E, Akmangit I, Kahilogullari G, Derakhshani S, Belen $D$, Dede D, Arat A: Woven low-profile stent as an alternative to dual stenting in bifurcation aneurysms. Interv Neuroradiol 19 Suppl 2:157, 2013
5. Darsaut TE, Bing F, Makoyeva A, Gevry G, Salazkin I, Raymond $\mathrm{J}$ : Flow diversion of giant curved sidewall and bifurcation experimental aneurysms with very-low-porosity devices. World Neurosurg 82:1120-1126, 2014

6. Darsaut TE, Bing F, Salazkin I, Gevry G, Raymond J: Flow diverters can occlude aneurysms and preserve arterial branches: A new experimental model. AJNR Am J Neuroradiol 33:2004-2009, 2012

7. Darsaut TE, Bing F, Salazkin I, Gevry G, Raymond J: Flow diverters failing to occlude experimental bifurcation or curved sidewall aneurysms: An in vivo study in canines. J Neurosurg 117:37-44, 2012

8. Darsaut TE, Rayner-Hartley E, Makoyeva A, Salazkin I, Berthelet F, Raymond J: Aneurysm rupture after endovascular flow diversion: The possible role of persistent flows through the transition zone associated with device deformation. Interv Neuroradiol 19:180-185, 2013

9. Estrade L, Makoyeva A, Darsaut TE, Ghostine J, Kouznetsov E, Salazkin I, Roy D, Weill A, Raymond J: In vitro reproduction of device deformation leading to thrombotic complications and failure of flow diversion. Interv Neuroradiol 19:432-437, 2013

10. Gurkas E, Kaya T, Daglioglu E, Akmangit I, Belen D, Peker A, Dede $D$, Arat A: Silk device for the treatment of intracranial aneurysms. Part 1: Periprocedural results, technical aspects and learning curve. Turk Neurosurg 26(4):525-532, 2016

11. Kaya T, Daglioglu E, Gurkas E, Akmangit I, Peker A, Belen D, Dede D, Elhan A, Arat A: Silk device for the treatment of intracranial aneurysms. Part 2: Factors related to clinical and angiographic outcome. Turk Neurosurg 26(4):533-537, 2016

12. Lubicz B, Van der Elst O, Collignon L, Mine B, Alghamdi F: Silk flow-diverter stent for the treatment of intracranial aneurysms: A series of 58 patients with emphasis on long-term results. AJNR Am J Neuroradiol 36:542-546, 2015

13. Makoyeva A, Bing F, Darsaut TE, Salazkin I, Raymond J: The varying porosity of braided self-expanding stents and flow diverters: An experimental study. AJNR Am J Neuroradiol 34:596-602, 2013

14. Raymond J, Darsaut TE, Makoyeva A, Bing F, Salazkin I: Endovascular treatment with flow diverters may fail to occlude experimental bifurcation aneurysms. Neuroradiology 55:13551363, 2013

15. Shapiro M, Raz E, Becske T, Nelson PK: Building multidevice pipeline constructs of favorable metal coverage: A practical guide. AJNR Am J Neuroradiol 35:1556-1561, 2014

16. Shapiro M, Raz E, Becske T, Nelson PK: Variable porosity of the pipeline embolization device in straight and curved vessels: A guide for optimal deployment strategy. AJNR Am J Neuroradiol 35:727-733, 2014

17. Topcuoglu OM, Akgul E, Daglioglu E, Topcuoglu ED, Peker A, Akmangit I, Belen D, Arat A: Flow diversion in middle cerebral artery aneurysms: Is it really an all-purpose treatment? World Neurosurg 87: 317-327, 2016 